\title{
Relationship between Formation of Geometrically Necessary Dislocations and Local Strain Hardening of Slip Systems in Symmetric Type Bicrystals under Tensile Loading *
}

\author{
Ryouji KONDOU ${ }^{* *}$ and Tetsuya OHASHI ${ }^{* * *}$ \\ ${ }^{* *}$ Faculty of Engineering, University of the Ryukyus, \\ 1 Senbaru, Nishihara-cho, Nakagami-gun, Okinawa 903-0213, Japan \\ E-mail: kondou@teada.tec.u-ryukyu.ac.jp \\ ${ }^{* * *}$ Kitami Institute of Technology, \\ 165 Koen-cho, Kitami-shi, Hokkaido 090-8507, Japan \\ E-mail: ohashi@newton.mech.kitami-it.ac.jp
}

\begin{abstract}
Slip deformation in symmetric-type bicrystal models subjected to tensile load is investigated by a finite element crystal plasticity analysis code. Accumulation of geometrically necessary dislocations (GNDs) and statistically stored dislocations (SSDs) are studied in detail. Some results of the analysis show local strain hardening of slip systems and activity of secondary slip systems with accumulation of GNDs on primary slip system in the form of band. Mechanism of local strain hardening of primary slip systems in symmetric-type bicrystals is discussed from the viewpoint of dislocation interaction between primary and secondary slip systems and effects of high density pattern formation of GNDs on primary slip system.
\end{abstract}

Key words: Crystal Plasticity Analysis, Geometrically Necessary Dislocation, Strain Hardening, Symmetric Type Bicrystal, Compatibility, Schmid Tensor

\section{Introduction}

When plastic deformation takes place in a polycrystalline metal, normally, in order to satisfy the continuity of displacement at the grain boundary, non-uniform deformation and accumulation of "geometrically necessary (GN) dislocations" (1) occurs near the grain boundary. From this, it has conventionally been thought that the main cause of the non-uniform deformation that takes place in a polycrystalline metal and the accumulation of GN dislocations are the deformation constraints that work interactively between crystal grains through the grain boundary due to the effects of the elastic anisotropy ${ }^{(2)}$ and the effect of the anisotropy of slip deformation.

We have performed crystal plasticity analysis of tensile deformation in symmetric-type bicrystals that satisfy the condition under which the deformation constraint does not work interactively between crystal grains through the grain boundary, that is, the so-called "compatible condition" (3) (4) of strain at the grain boundary, and stated in a previous study ${ }^{(5)}$ that there may be cases where non-uniform deformation takes place. At the same time, GN dislocations accumulate in a high density (the structure in which GN dislocations thus accumulate has been called the "GN dislocation band") in a band-like area that has developed in the inward direction of the crystal grain, even in the symmetric-type bicrystals, depending on how the load is applied. It has thus been clarified that the cause of the non-uniform deformation that takes place in a polycrystalline metal - the occurrence of the GN dislocation and the structure formation of the GN dislocation - is not necessarily the 
deformation constraint that works interactively between crystal grains through the grain boundary. Therefore, it is necessary to evaluate the influence of the boundary conditions, such as the load condition, the grain boundary plane, the free surface, etc., on the slip deformation of the crystal grain.

When non-uniform deformation takes place in the crystal grain, microscopic stress concentration occurs, the primary stress direction deviates from the load direction, and an increase/decrease in the critical resolved shear stress in the primary slip system and the secondary slip system results ${ }^{(6)}$. Further, in the GN dislocation, the attenuation in stress is less than that in a dislocation which is a positive-negative pair due to the positive-negative imbalance, and an inner stress field (back stress) extending in a long range is formed ${ }^{(7)}$. Consequently, when a structure based on GN dislocations like the "GN dislocation band" which is accumulated in a high density in a local area in the crystal grain is formed, it can be considered that an extremely significant influence is imposed on the after-deformation behavior.

This study examined the structure formation of the "GN dislocation band" accompanying tensile deformation in symmetric-type bicrystals. To clarify the evaluation method and the influence exerted on the after-deformation state, crystal plasticity analysis of tensile deformation of various symmetric type bicrystals having different initial crystal orientations was performed, and the change in the distribution of the GN dislocation density was discussed.

\section{Analysis Method}

\section{2:1 Analysis of deformation by finite element method}

If it is assumed that the activation condition of the slip system is given by the Schmid law, the following equation will hold between the stress tensor $\sigma_{i j}$ under the activation condition and the critical resolved shear stress $\theta^{(n)}$ in the $n$-th slip system:

$$
\begin{aligned}
& P_{i j}^{(n)} \sigma_{i j}=\theta^{(n)} \\
& P_{i j}^{(n)} \dot{\sigma}_{i j}=\dot{\theta}^{(n)} \\
& P_{i j}^{(n)}=\frac{1}{2}\left\{v_{i}^{(n)} b_{j}^{(n)}+v_{j}^{(n)} b_{i}^{(n)}\right\}
\end{aligned}
$$

where, $P_{i j}^{(n)}$ is the Schmid tensor in the $n$-th slip system, wherein the unit vector in the plane normal direction is $v_{i}^{(n)}$ and the unit vector in the slip direction is $b_{i}^{(n)}$.

The strain increment $\dot{\varepsilon}_{i j}$ is acquired from the sum of the elastic component $\dot{\varepsilon}_{i j}^{e}$ and the plastic component $\dot{\varepsilon}_{i j}^{p}$ using the following equation:

$$
\begin{aligned}
& \dot{\varepsilon}_{i j}=\dot{\varepsilon}_{i j}^{e}+\dot{\varepsilon}_{i j}^{p} \\
& \dot{\varepsilon}_{i j}^{e}=S_{i j k l}^{e} \dot{\sigma}_{k l} \\
& \dot{\varepsilon}_{i j}^{p}=\sum_{n} \dot{\gamma}^{(n)} P_{i j}^{(n)}
\end{aligned}
$$

where, $S_{i j k l}^{e}$ is an elastic compliance and $\dot{\gamma}^{(n)}$ is a plastic shear strain increment.

If it is assumed that the strain hardening coefficient is $h^{(n m)}$ and the strain hardening law is represented by the following equation:

$$
\dot{\theta}^{(n)}=\sum_{m} h^{(n m)} \dot{\gamma}^{(m)}
$$

then the elastic/plastic constitutive equation ${ }^{(8)}$ is represented by the following equation:

$$
\dot{\sigma}_{i j}=\left[S_{i j k l}^{e}+\sum_{n} \sum_{m}\left\{h^{(n m)}\right\}^{-1} P_{i j}^{(n)} P_{k l}^{(m)}\right]^{-1} \dot{\varepsilon}_{k l}
$$

However, $n$ and $m$ are added to obtain their sum only in active slip systems. Based on the elastic/plastic constitutive equation, the deformation of the model is analyzed using the finite element method.

\section{2-2 Analysis of dislocation density}


Dislocations present inside the material having been subjected to deformation hysteresis are roughly classified into two kinds, that is, "statistically stored dislocations" (SS dislocations) and GN dislocations, and their density is calculated from the strain increment, which is the result of analyzing deformation. The relationship between the increment $\dot{\rho}_{S}^{(n)}$ of the SS dislocation density and $\dot{\gamma}^{(n)}$ is represented by the following equation ${ }^{(9)}$ :

$$
\dot{\rho}_{S}^{(n)}=\frac{c \dot{\gamma}^{(n)}}{\tilde{b} L^{(n)}}
$$

where, $c$ is a coefficient of the order of $1, \tilde{b}$ is the magnitude of the Burgers vector, and $L^{(n)}$ is an mean free path of dislocation.

The slip deformation of the crystal grain is produced by the motion of dislocations, and therefore, in the area in which dislocations have passed, slip deformation occurs and in the area in which dislocations have not passed yet, no slip deformation occurs. Consequently, at a place where a spatial gradient of $\gamma^{(m)}$ is produced, dislocations must exist. This is called the GN dislocation ${ }^{(1)}$.

If it is assumed that the direction parallel to the slip direction on the slip plane is $\xi^{(m)}$, the direction perpendicular to the slip direction on the slip plane is $\zeta^{(m)}$, and as to the spatial gradient of $\gamma^{(m)}$, the component in the direction parallel to the slip direction is an edge dislocation density component $\rho_{G, e d g e}^{(m)}$, and the component in the direction perpendicular to the slip direction is a screw dislocation density component $\rho_{G \text {,screw }}^{(m)}$, then the norm of the GN dislocation density $\left\|\rho_{G}^{(m)}\right\|$ is represented by the following equation ${ }^{(10)}$ :

$$
\begin{aligned}
\left\|\rho_{G}^{(m)}\right\| & =\sqrt{\left(\rho_{G, \text { edge }}^{(m)}\right)^{2}+\left(\rho_{G, \text { screw }}^{(m)}\right)^{2}} \\
\rho_{G, \text { edge }}^{(m)} & =-\frac{1}{\tilde{b}} \frac{\partial \gamma^{(m)}}{\partial \xi^{(m)}} \\
\rho_{G, \text { screw }}^{(m)} & =\frac{1}{\tilde{b}} \frac{\partial \gamma^{(m)}}{\partial \zeta^{(m)}}
\end{aligned}
$$

\section{2-3 Deformation hysteresis and evaluation method}

The critical resolved shear stress $\theta^{(n)}$ in the slip system in a face-centered cubic crystal having been subjected to deformation is represented by the modified Bailey-Hirsch equation $^{(9)}$ :

$$
\theta^{(n)}=\theta_{0}(T)+\sum_{m=1}^{12} a \mu \tilde{b} \Omega^{(n m)} \sqrt{\rho_{s}^{(m)}}
$$

where, $\theta_{0}$ is a resistance value that does not depend on the deformation hysteresis against the moving dislocation, $a$ is a numerical value of about $0.1, \mu$ is an elastic shear modulus of crystal, and $\Omega^{(\mathrm{nm})}$ is an interactive matrix ${ }^{(9)} . \quad \Omega^{(\mathrm{nm})}$ indicates the strength of damage of the dislocations accumulated on the slip system imposed on the moving dislocation on another slip system, and can be represented by five kinds of parameters $R_{1}, R_{2}, R_{3}, R_{3}$ ', and $R_{4}$, the interactive strength between the dislocations on the same slip system being the reference unit. Here, isotropic hardening is supposed and so $R_{1}=R_{2}=R_{3}=R_{3}{ }^{\prime}=R_{4}=1.01$. This equation simplifies the multi-body interaction (thought to be extremely complex) that works between the moving dislocation and the stored dislocation into an abstract image in which the dislocation moves in the isotropic homogeneous field formed by the stored dislocations.

The hardening coefficient $h^{(\mathrm{nm})}$ of the strain of the material having been subjected to the deformation hysteresis is represented by the following equation:

$$
h^{(n m)}=\frac{1}{2} \frac{a c \mu \Omega^{(n m)}}{L^{(m)} \sqrt{\rho_{S}^{(m)}}}
$$

As a method of calculating the mean free path $L^{(n)}$ in equations (5) and (8), a dislocation density-dependent type model ${ }^{(11)(12)}$, in which the SS dislocation and the GN dislocation contribute, is used. 


$$
L^{(n)}=\frac{c^{*}}{\sqrt{\sum_{m} \omega^{(n m)}\left(\rho_{S}^{(m)}+\left\|\rho_{G}^{(m)}\right\|\right)}}
$$

This model is based on an abstract image that the moving dislocation comes to a stop after having moved the distance $c^{*}$ times the average interval of the accumulated dislocations. Here, $c^{*}=15$. $\omega^{(n m)}$ is a weighting matrix ${ }^{(11)(12)}$, which represents the interaction between the moving dislocation and the stored dislocation, and can be represented by six kinds of parameters $w_{0}, w_{1}, w_{2}, w_{3}, w_{3}{ }^{\prime}$, and $w_{4}$. Here, considering that dislocations stored in the self-slip system and the common plane slip system do not contribute to the capture of the moving dislocations, it is assumed that $w_{0}=w_{1}=0$, and $w_{2}=$ $w_{3}=w_{3}{ }^{\prime}=w_{4}=1.0$.

Due to the contribution of the density of GN dislocations having size dependence on $L^{(n)}$, the GN dislocations concern the accumulation of the SS dislocations (refer to equation (5)), and the size effect occurs in the critical resolved shear stress and the strain hardening characteristics (refer to equations (7) and (8)).

\subsection{Increment analysis}

The present analysis consists of three parts, that is, the analysis of deformation (section 2.1 ), the calculation of the dislocation density (section 2.2), and the evaluation of strain hardening in the slip system (section 2.3).

At first, the constitutive equation of elastic deformation is determined from the elastic compliance and the initial crystal orientation, and the elastic deformation up to immediately before yield point in accordance with the boundary condition is analyzed. Next, an active slip system is selected by the Schmid law, a strain hardening coefficient is determined from the initial dislocation density, etc., the constitutive equation is modified, and the slip deformation is analyzed. Since strain hardening occurs in the slip system as deformation advances, it is necessary to dynamically modify the constitutive equation. To analyze the non-linear phenomenon, the increment analysis is performed by dividing time into steps each time the slip system starts to act and terminates. In other words, the SS dislocation density $\rho_{S}^{(m)}$ and the GN dislocation density norm $\left\|\rho_{G}^{(m)}\right\|$ serve as internal state variables for determining the constitutive equation in the next time step.

\section{Model}

The analysis model is symmetric-type bicrystals as shown in Fig. 1, and the length $l$ of a side is $200 \mu \mathrm{m}$, and the thickness $t$ is $10 \mu \mathrm{m}$. The grain boundary plane is perpendicular to the model surface. Finite element division is carried out uniformly using eight-node hexahedron elements. The total number of elements is 3,200 .

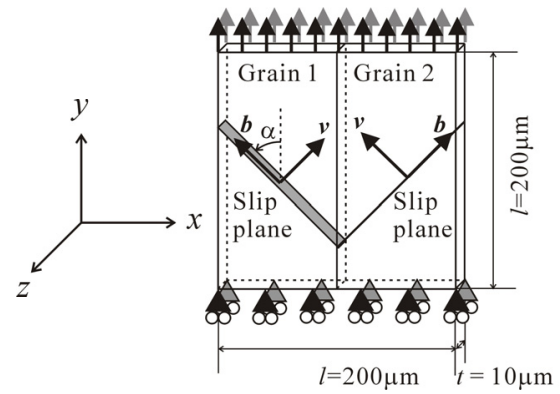

Fig. 1 Geometry and boundary condition for the models employed in this study

When grains 1 and 2 deform independently of each other and if their combination is such that it produces a difference between the strain components in the direction in the grain boundary plane, deformation constraint occurs between the crystal grains when grains 1 and 2 couple with each other and deform. The condition under which interaction of such 
deformation constraint is not produced is called the "compatible condition" (3) (4). Note that here the compatible condition is different in meaning from the compatible condition in solid-state physics. In this case, it is given by the following expression:

$$
\varepsilon_{y y}^{(1)}=\varepsilon_{y y}^{(2)}, \varepsilon_{z z}^{(1)}=\varepsilon_{z z}^{(2)}, \quad \varepsilon_{y z}^{(1)}=\varepsilon_{y z}^{(2)}
$$

where, $\varepsilon_{y y}^{(1)}, \varepsilon_{y y}^{(2)}$, etc., are strain components (the sum of the elastic strain component and the plastic strain component) produced when grains 1 and 2 deform independently of each other, and the superscripts denotes the crystal grain numbers.

A virtual face-centered cubic crystal metal is used as a material and in order to prevent an elastic incompatible stress due to the effect of elastic anisotropy ${ }^{(2)}$ from being produced, it is assumed that the combinations of the elastic compliances of the standard crystal orientation are $S_{11}=1.0 \times 10^{-11}, S_{12}=-0.25 \times 10^{-11}$, and $S_{44}=2.5 \times 10^{-11}\left[\mathrm{~m}^{2} / \mathrm{N}\right]$ and so the elastic anisotropic ratio $2\left(S_{11}-S_{12}\right) / S_{44}$ is 1 .

The relationship between the crystal coordinate system constituted by the unit vectors [100], [010], and [001] and the material coordinate system $(x, y, z)$ is defined by Euler angles $(\kappa, \theta, \phi)$ shown in Fig. 2.

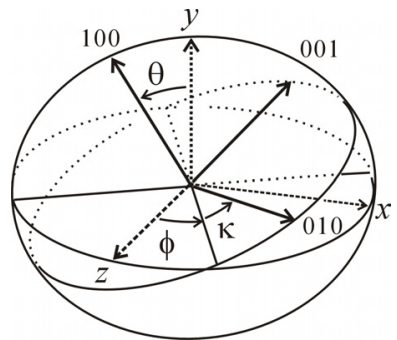

Fig. 2 Definition of Euler angles $\kappa, \theta$ and $\phi$

There are several methods of defining Euler angles. By Euler angles, the coordinate transformation matrix between the material coordinate system and the crystal coordinate system is given by the following expression:

$$
\left(\begin{array}{l}
{[100]} \\
{[010]} \\
{[001]}
\end{array}\right)=\left(\begin{array}{ccc}
-\sin \theta \cos \phi & \cos \theta & \sin \theta \sin \phi \\
\cos \kappa \sin \phi+\cos \theta \sin \kappa \cos \phi & \sin \theta \sin \kappa & \cos \kappa \cos \phi-\cos \theta \sin \kappa \sin \phi \\
-\sin \kappa \sin \phi+\cos \theta \cos \kappa \cos \phi & \sin \theta \cos \kappa & -\sin \kappa \cos \phi-\cos \theta \cos \kappa \sin \phi
\end{array}\right)\left(\begin{array}{l}
x \\
y \\
z
\end{array}\right)(11)
$$

It is assumed that the initial crystal orientation of grain 1 has 11 orientations as shown in Table 1, and the initial crystal orientation of grain 2 is one when grain 1 is rotated about the $y$-axis through 180 degrees.

Table 1 Euler angle $(\kappa, \theta, \phi)$, angle between slip direction $b^{(9 t h)}$ and tensile loading direction $\alpha$,components of slip direction vector $b^{(9 t h)}$, slip plane normal vector $v^{(9 t h)}$ and value of Schmid tensor $\boldsymbol{P}_{i j}^{(9 t h)},\left(v_{z}^{(9 t h)}=b_{z}^{(9 t h)}=\mathbf{0}, P_{33}^{(9 t h)}=P_{23}^{(9 t h)}=P_{31}^{(9 t h)}=\mathbf{0}\right)$

\begin{tabular}{|c|l|l|l|l|l|l|l|l|}
\hline$\kappa, \theta, \phi[\mathrm{deg}]$ & $\begin{array}{l}\alpha \\
{[\mathrm{deg}]}\end{array}$ & $b_{x}^{(9 t h)}$ & $b_{y}^{(9 t h)}$ & $v_{x}^{(9 t h)}$ & $v_{y}^{(9 t h)}$ & $P_{11}^{(9 t h)}$ & $P_{22}^{(9 t h)}$ & $P_{12}^{(9 t h)}$ \\
\hline $74.983,24.535,79.470$ & 44.0 & -0.6947 & 0.7193 & 0.7193 & 0.6947 & -0.49970 & 0.49970 & 0.01745 \\
\hline $75.456,24.572,79.040$ & 44.2 & -0.6972 & 0.7169 & 0.7169 & 0.6972 & -0.49981 & 0.49981 & 0.01396 \\
\hline $75.927,24.611,78.611$ & 44.4 & -0.6997 & 0.7145 & 0.7145 & 0.6997 & -0.49989 & 0.49989 & 0.01047 \\
\hline $76.397,24.650,78.183$ & 44.6 & -0.7022 & 0.7120 & 0.7120 & 0.7022 & -0.49995 & 0.49995 & 0.00698 \\
\hline $76.866,24.692,77.757$ & 44.8 & -0.7046 & 0.7096 & 0.7096 & 0.7046 & -0.49999 & 0.49999 & 0.00349 \\
\hline $77.333,24.735,77.333$ & 45.0 & -0.7071 & 0.7071 & 0.7071 & 0.7071 & -0.50000 & 0.50000 & 0.00000 \\
\hline $77.799,24.780,76.910$ & 45.2 & -0.7096 & 0.7046 & 0.7046 & 0.7096 & -0.49999 & 0.49999 & -0.00349 \\
\hline $78.263,24.826,76.489$ & 45.4 & -0.7120 & 0.7022 & 0.7022 & 0.7120 & -0.49995 & 0.49995 & -0.00698 \\
\hline $78.725,24.873,76.070$ & 45.6 & -0.7145 & 0.6997 & 0.6997 & 0.7145 & -0.49989 & 0.49989 & -0.01047 \\
\hline $79.186,24.922,75.652$ & 45.8 & -0.7169 & 0.6972 & 0.6972 & 0.7169 & -0.49981 & 0.49981 & -0.01396 \\
\hline $79.645,24.972,75.236$ & 46.0 & -0.7193 & 0.6947 & 0.6947 & 0.7193 & -0.49970 & 0.49970 & -0.01745 \\
\hline
\end{tabular}


These orientations are $v_{z}^{(9 t h)}=b_{z}^{(9 t h)}=0$ as to the primary slip system (11 $\left.\overline{1}\right)[101]$ and the angle $\alpha$ formed by the slip direction $\boldsymbol{b}^{(9 t h)}$ and the direction of load is $44^{\circ}$ to $46^{\circ}$. In this case, the value of the Schmid factor of the primary slip system (11 $\overline{1})[101]$ is about 0.5 ; and under minute deformation, the deformation of the model advances due to the single slip in the primary slip system.

As $v_{z}^{(9 t h)}=b_{z}^{(9 t h)}=0$, the values of the Schmid tensors $P_{33}^{(9 t h)}, P_{23}^{(9 t h)}, P_{31}^{(9 t h)}$ in grains 1 and 2 are 0 and the plastic strain components $\varepsilon_{z z}^{p}, \varepsilon_{y z}^{p}, \varepsilon_{z x}^{p}$ that occur in grains 1 and 2 are 0 (refer to equation (2)). Since the orientation of grain 2 is one when grain 1 is rotated about the y-axis through 180 degrees, the result is that only the signs of $v_{x}^{(9 t h)}$ and $b_{x}^{(9 t h)}$ are opposite, and the values of $v_{y}^{(9 t h)}$ and $b_{y}^{(9 t h)}$ are equal, and the value of $P_{22}^{(9 t h)}$ is equal, and $\varepsilon_{y y}^{p}$ that occurs in grains 1 and 2 is equal, satisfying the compatible conditional expression (10).

It is assumed that the initial dislocation density $\rho_{0}$ is uniform, $1.0 \times 10^{9}\left[\mathrm{~m}^{-2}\right]$.

The displacement in the $y$-axis direction at all the nodes on the bottom plane of the model is constrained, a uniform forced displacement in the y-axis direction is imposed on all the nodes on the top plane, and pulling is performed until the average tensile strain $\bar{\varepsilon}_{y y}$ becomes $1 \%$.

\section{Analysis Results and Consideration}

\section{4 - 1 Differences in the "GN dislocation band" depending on the initial crystal orientation}

We previously reported ${ }^{(5)}$ that when tensile caused by forced displacement is given to symmetric type bicrystals, even if the compatible condition is satisfied, there may be cases where non-uniform deformation takes place; and at the same time, GN dislocations accumulate in a high density in a band-like area that has developed in the crystal in an inward direction from the vicinity of the intersection of the model upper plane (or bottom plane) and the grain boundary plane. This GN dislocation structure is called "GN dislocation band." In this case, the "GN dislocation band" is formed irrespective of the sizes of the crystal grain and the strain hardening characteristics and is constituted by the almost perfect edge dislocation density component $\rho_{G, \text { edge }}^{\left({ }^{(5)}\right)}$. In this study, the differences in the "GN dislocation band" depending on the initial crystal orientation, and a method of evaluating them, are described.

The distribution of the edge dislocation density components $\rho_{G, \text { edge }}^{(9 t h)}$ of the GN dislocation in the primary slip system $(11 \overline{1})[101]$ in the stage at which the average tensile strain $\bar{\varepsilon}_{y y}$ is $0.01 \%$ is shown in Fig. 3.

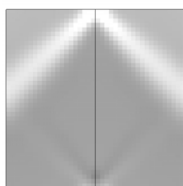

(a) $\alpha=44.0^{\circ}$

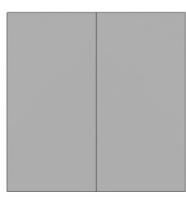

(f) $\alpha=45.0^{\circ}$

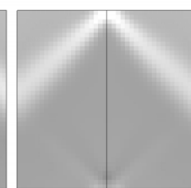

(b) $\alpha=44.2^{\circ}$

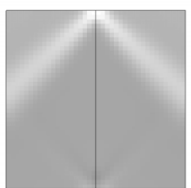

(c) $\alpha=44.4^{\circ}$

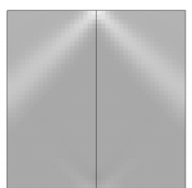

(d) $\alpha=44.6^{\circ}$

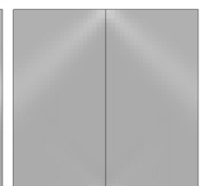

(e) $\alpha=44.8^{\circ}$

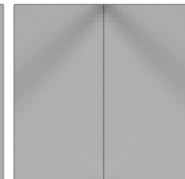

(g) $\alpha=45.2^{\circ}$

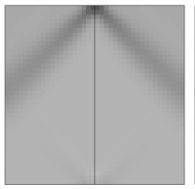

(h) $\alpha=45.4^{\circ}$

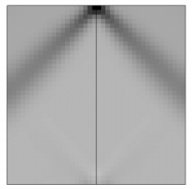

(i) $\alpha=45.6^{\circ}$

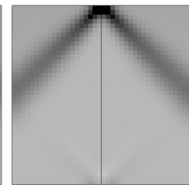

(j) $\alpha=45.8^{\circ}$

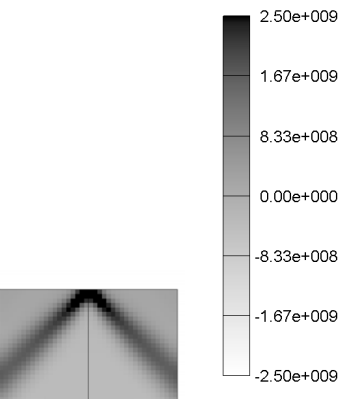

(k) $\alpha=46.0^{\circ}$

Fig. 3 Density distribution of edge components of geometrically necessary dislocations on primary slip system when average tensile strain $\bar{\varepsilon}_{y y}$ is $0.01 \%$.

It is confirmed that the model deforms due to a single slip of the primary slip system in each case up to this strain stage. Here, in order to compare the difference of the "GN 
dislocation band" depending on the initial crystal orientation (angle $\alpha$ ), the display range of the GN dislocation density is fixed to $-2.5 \times 10^{9}$ to $2.5 \times 10^{9}\left[\mathrm{~m}^{-2}\right]$.

When the angle $\alpha$ is $45^{\circ}$ (Fig. 3(f)), no GN dislocations occur; and when the angle $\alpha$ deviates from $45^{\circ}$, in either of the cases, that is, both on the lower angle side from $45^{\circ}$ (Figs. $3(\mathrm{a})$ to (e)) and on the higher angle side (Figs. $3(\mathrm{~g})$ to (k)), a structure in which GN dislocations accumulate in a high density in a band-like area that has developed in the crystal in an inward direction from the vicinity of the intersection of the model upper plane and the grain boundary, that is, the "GN dislocation band," is formed. The density and the sign of the "GN dislocation band" differ depending on the angle $\alpha$, and it is known that the further the angle $\alpha$ deviates from $45^{\circ}$, the higher the density of accumulated dislocations becomes. Further, it is also known that the signs of the GN dislocations that form the "GN dislocation band" are opposite between the lower angle side from $45^{\circ}$ (Figs. 3(a) to (e)) and the higher angle side (Figs. 3(g) to (k)).

\section{2 Relationship between Schmid tensor value and "GN dislocation band"}

When the boundary condition is imposed so that uniform deformation takes place on the upper and bottom planes of the symmetric-type bicrystal model, the relationship of perpendicularity between the upper and bottom planes of the model and the grain boundary plane must be maintained also after deformation; and therefore, the change in the shape of grains 1 and 2 due to the slip deformation in the primary slip system is constrained by the upper and bottom planes of the model and the grain boundary plane. Since the plastic strain increment $\dot{\varepsilon}_{x y}^{p}$ accompanying the slip in the primary slip system of grains 1 and 2 depends on the value of the Schmid tensor $P_{12}^{(9 t h)}$ (refer to equation (2)), the constrained plastic strain component also depends on the value of $P_{12}^{(9 t h)}$. The value and sign of $P_{12}^{(9 t h)}$ depend on the crystal orientation and in this case, the signs of the angle $\alpha$ are opposite between the lower angle side from $45^{\circ}$ and the higher angle side (refer to Table 1); and therefore, the signs of the GN dislocations that have formed the "GN dislocation band" are also opposite between the lower angle side where the angle $\alpha$ is lower than $45^{\circ}$ (Fig. 3(a) to (c)) and the higher angle side (Fig. 3(g) to (k)).

When the GN dislocation does not occur (Fig. 3(f)), it means that no constraint acts on the change in the shape of grains 1 and 2 due to the free slip deformation in the primary slip system. This condition is satisfied when the plastic shear strain increment $\dot{\varepsilon}_{x y}^{p}$ of grains 1 and 2 is 0 , that is, the value of $P_{12}^{(9 t h)}$ is 0 (angle $\alpha$ is $45^{\circ}$ ).

The relationship between the value of $P_{12}^{(9 t h)}$ in each strain stage up to $0.01 \%$ of the average tensile strain $\bar{\varepsilon}_{y y}$ and the average value $\overline{\left\|\rho_{G}^{(9) i}\right\|}$ in all the elements of the GN dislocation density norm $\overline{\left\|\rho_{G}^{(9, h)}\right\|}$ is shown in Fig. 4(a), and its relationship with the standard deviation $S$ is shown in Fig. 4(b).
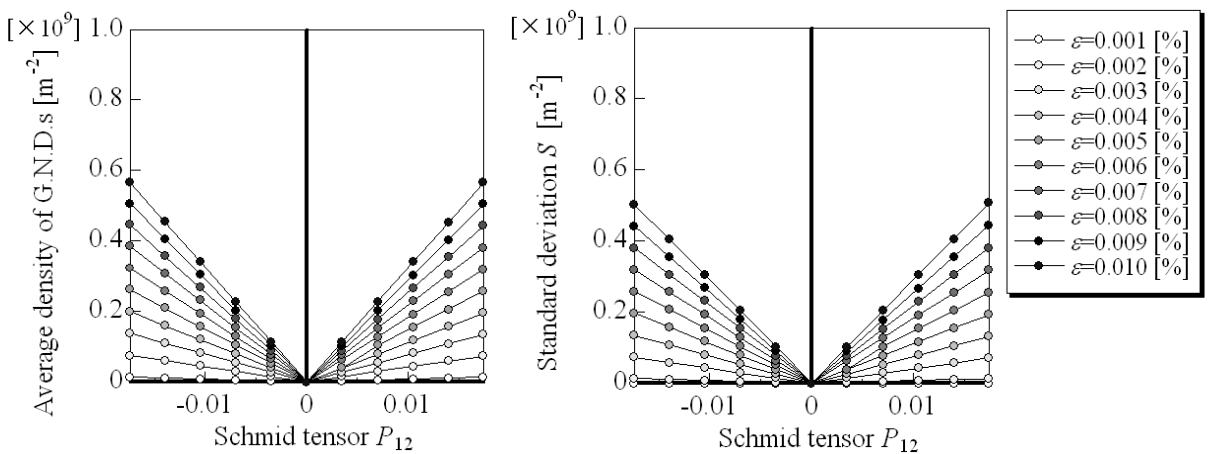

Fig. 4 (a) Averaged value of density of norm of geometrically necessary dislocations and (b) its standard deviation $S$, plotted against Schmid tensor $P_{12}$

It is known that while the deformation of the model is advancing due to the single slip in the primary slip system, the density of GN dislocations that accumulate increases linearly 
in accordance with the increase in the absolute value $\left\|P_{12}^{(9 t h)}\right\|$ of $P_{12}^{(9 t h)}$. It is also known that the increment of the density of GN dislocations accompanying the increase of $\bar{\varepsilon}_{y y}$ increases, depending on $\left\|P_{12}^{(9 t h)}\right\|$.

It is known that the value of the standard deviation $S$ increases linearly as $\left\|P_{12}^{(9 t h)}\right\|$ increases, and the non-uniformity of the distribution of the density of GN dislocations develops. It is also known that the development of $S$ accompanying the increase in $\bar{\varepsilon}_{y y}$ becomes remarkable depending on $\left\|P_{12}^{(9 t h)}\right\|$. The development of $S$ means that GN dislocations accumulate in a local area, that is, the formation of the structure of GN dislocations. In this case, it has been found that the density of GN dislocations constituting the "GN dislocation band" can be evaluated by the value of the Schmid tensor.

\section{4 -3 Relationship between "GN dislocation band" and strain hardening in slip system}

Since the GN dislocation is due to the positive-negative imbalance, in the stress field formed by this, attenuation is less than in the stress field formed by the dislocation in which a positive-negative pair is made and extends in a longer range ${ }^{(7)}$. Further, when the slip occurs non-uniformly, microscopic stress concentration occurs, the primary stress axis deviates from the load direction, and an increase/decrease in the critical resolved shear stress in the primary slip system and the secondary slip system results ${ }^{(6)}$. Consequently, as in the "GN dislocation band," when the GN dislocations having the same sign accumulate in a high density in a local area, it can be thought that an extremely strong inner stress field is formed and at the same time, the secondary slip system in the local area is activated. Here, the difference of the state of deformation is described, between the stage at which the average tensile strain $\bar{\varepsilon}_{y y}$ is $0.1 \%$ and the stage at which pulling is performed until it becomes $1 \%$ in the model in which the angle $\alpha$, with which the high density "GN dislocation band" has been formed, is set to $44^{\circ}$ and $46^{\circ}$. For the plastic shear strain and the SS dislocation density, the value from the maximum to the minimum is displayed; and for the GN dislocation density, the display range is fixed for comparison.

It is known that, as shown in Figs. 5(e) and $(\mathrm{g})$, resulting from the formation of the structure of the "GN dislocation band" of the primary slip system (11) $[101]$, the secondary slip systems (111)[10 $\overline{1}]$ and (1 $\overline{1} 1)[110]$ are activated in an extremely local area in the vicinity of the "GN dislocation band." Incidentally, when the amount of the plastic shear strains $\gamma^{(3 r d)}$ and $\gamma^{(10 t h)}$ that have occurred in the secondary slip system are compared with the amount of the plastic shear strain $\gamma^{(9 t h)}$ that has occurred in the primary slip system (Fig. 5(a)), the result is as slight as about 1/1,000 to $1 / 100$; however, the strains have occurred in a local area, and therefore, a steep spatial gradient is produced, and the GN dislocations accumulate. In particular, the accumulation of $\left\|\rho_{G}^{(10 t h)}\right\|$ is remarkable (Fig. $5(\mathrm{~h}))$.

In the area in which the GN dislocations have accumulated in the secondary slip system, due to the dislocation interaction, the mean free path of the moving dislocation is reduced (refer to equation (9)); and therefore, in this area, the density of SS dislocations in the primary slip system rises abruptly (refer to equation (5)), causing strain hardening in the local primary slip system to occur (refer to equation (8)). Because of this, the distribution aspects differ between the plastic shear strain $\gamma^{(9 t h)}$ in the primary slip system and the SS dislocation density $\rho_{S}^{(9 t h)}$ (Fig. 5(a) and (b)). In addition, due to the dislocation interaction, irrespective of $v_{z}^{(9 t h)}=b_{z}^{(9 t h)}=0$, a spatial gradient of $\gamma^{(9 t h)}$ occurs also in the direction of thickness and the screw dislocation density component $\rho_{G, \text { screw }}^{(9 t)}$ is produced (Fig. 5(d)).

Since the spatial arrangement of the primary slip system and the secondary slip system depends on the crystal orientation, the kind and the position of the activated secondary slip system differ depending on the angle $\alpha$ (crystal orientation) (Fig. 6). When the angle $\alpha$ is $46^{\circ},(1 \overline{1} 1)[110]$ is not activated (both the maximum value and the minimum value of 
$\gamma^{(10 t h)}$ are 0) (Fig. 6(g)), and therefore, the deformation of the model advances only by the slip deformation in the primary slip system and the secondary slip system (111)[10 $\overline{1}]$.

As a result, the density of GN dislocations that accumulates in the secondary slip system

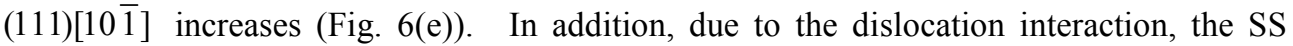
dislocation components that accumulate in the primary slip system (Fig. 6(b)) and the screw dislocation components of the GN dislocations (Fig. 6(d)) also accumulate in a high density, and the non-uniformity of the slip deformation in the primary slip system develops remarkably.

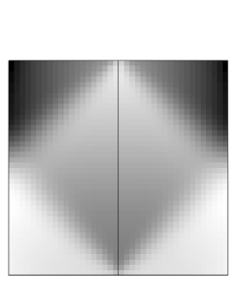

(a) $\gamma^{(9 t h)}$

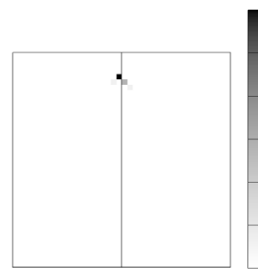

(e) $\gamma^{(3 r d)}$

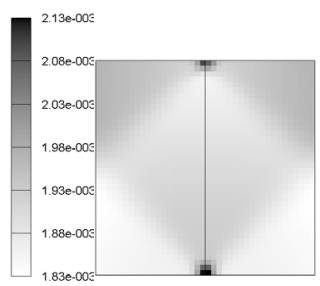

(b) $\rho_{S}^{(9 t h)}$

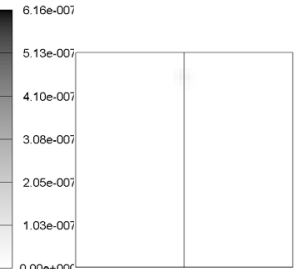

(f) $\left\|\rho_{G}^{(3 r d)}\right\|$

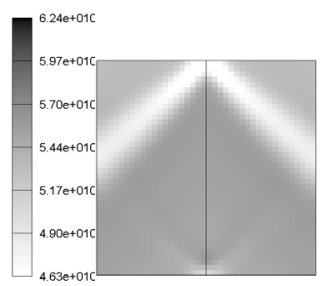

(c) $\rho_{G, e d g e}^{(9 t h)}$

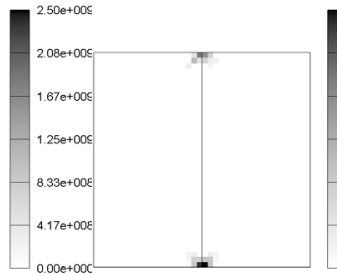

(g) $\gamma^{(10 t h)}$

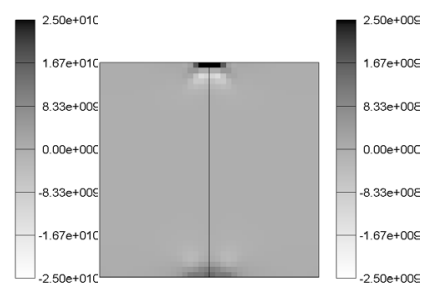

(d) $\rho_{G, s c r e w}^{(9 t h)}$

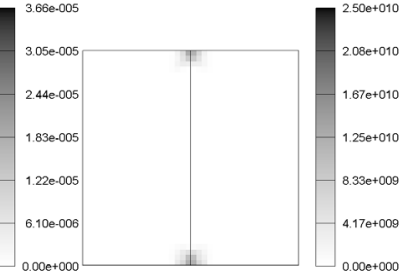

(h) $\left\|\rho_{G}^{(10 t h)}\right\|$

Fig. 5 (a) Distribution of plastic shear strain, (b) density distribution of statistically stored dislocations, (c) edge and (d) screw components of geometrically necessary dislocations on the primary slip system, (e), (g) distribution of plastic shear strain and (f), (h) density of norm of geometrically necessary dislocations on (111)[10 $\overline{1}]$ and (1)1)[110] slip systems when the average tensile strain $\bar{\varepsilon}_{y y}$ is $0.1 \%$ at the bicrystal model of $\alpha=44^{\circ}$. The unit of dislocation density is $\mathrm{m}^{-2}$

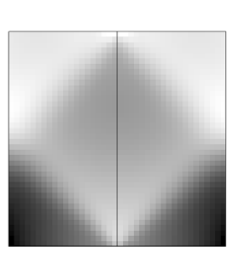

(a) $\gamma^{(9 t h)}$

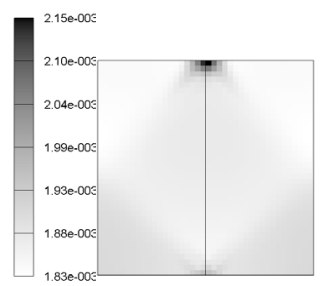

(b) $\rho_{S}^{(9 t h)}$

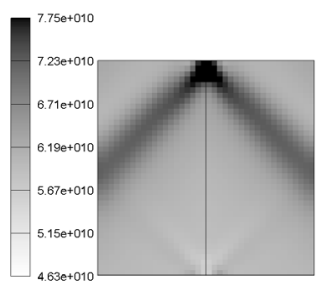

(c) $\rho_{G, e d g e}^{(9 t h)}$

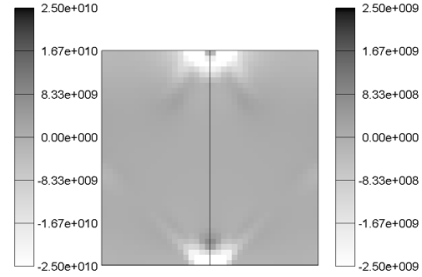

(d) $\rho_{G, s c r e w}^{(9 t h)}$

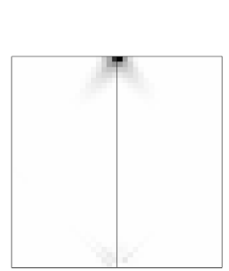

(e) $\gamma^{(3 r d)}$

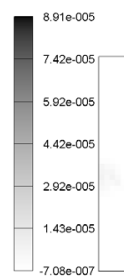

(f) $\| \rho_{G}^{(3 r d)}$

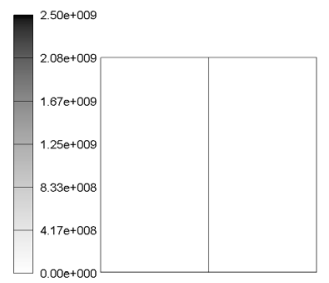

(g) $\gamma^{(10 t h)}$

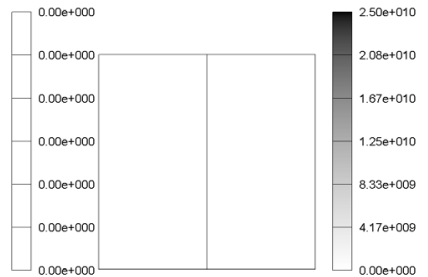

(h) $\left\|\rho_{G}^{(10 t h)}\right\|$

Fig. 6 (a) Distribution of plastic shear strain, (b) density distribution of statistically stored dislocations, (c) edge and (d) screw components of geometrically necessary dislocations on the primary slip system, (e), (g) distribution of plastic shear strain and (f), (h) density of norm of geometrically necessary dislocations on (111)[10 $\overline{1}]$ and $(1 \overline{1} 1)[110]$ slip systems when the average tensile strain $\bar{\varepsilon}_{y y}$ is $0.1 \%$ at the bicrystal model of $\alpha=46^{\circ}$. The unit of dislocation density is $\mathrm{m}^{-2}$ 
The difference of the state of deformation depending on the angle $\alpha$ (crystal orientation) in the stage where deformation has advanced (the average tensile strain $\bar{\varepsilon}_{y y}=1 \%$ ) is shown in Fig. 7 and Fig. 8. Incidentally, the display range of the GN dislocation density is enlarged by a factor of 10 compared to that in Fig. 5 and Fig. 6.

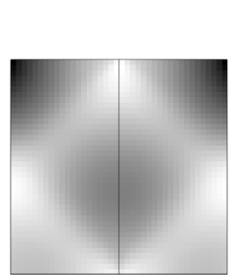

(a) $\gamma^{(9 t h)}$

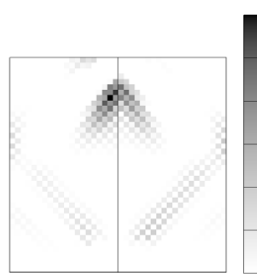

(e) $\gamma^{(3 r d)}$

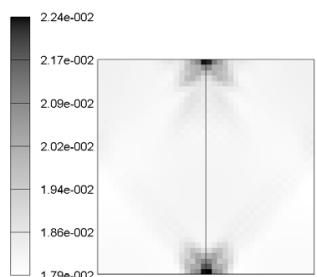

(b) $\rho_{S}^{(9 t h)}$

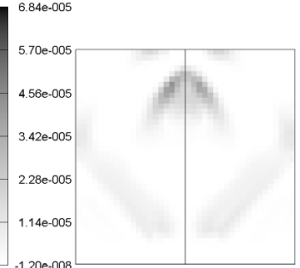

(f) $\left\|\rho_{G}^{(3 r d)}\right\|$

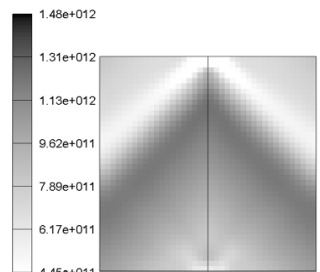

(c) $\rho_{G, e d g e}^{(9 t h)}$

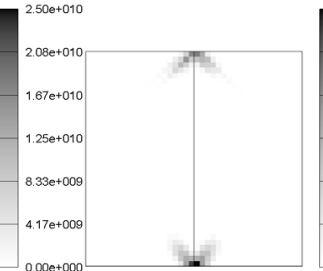

(g) $\gamma^{(10 t h)}$

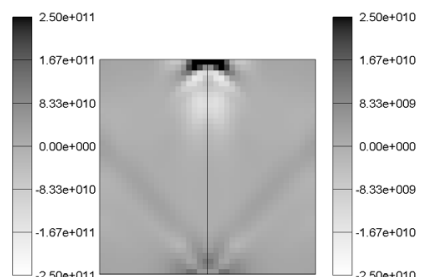

(d) $\rho_{G, s c r e w}^{(9 t h)}$

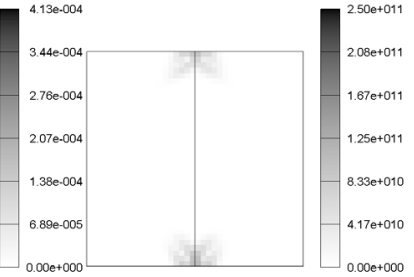

(h) $\left\|\rho_{G}^{(10 t h)}\right\|$

Fig. 7 (a) Distribution of plastic shear strain, (b) density distribution of statistically stored dislocations, (c) edge and (d) screw components of geometrically necessary dislocations on the primary slip system, (e), (g) distribution of plastic shear strain and (f), (h) density of norm of geometrically necessary dislocations on (111)[10 $\overline{1}]$ and (111)[110] slip systems when the average tensile strain $\bar{\varepsilon}_{y y}$ is $1 \%$ at the bicrystal model of $\alpha=44^{\circ}$. The unit of dislocation density is $\mathrm{m}^{-2}$

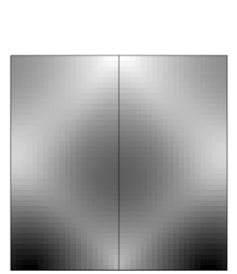

(a) $\gamma^{(9 t h)}$

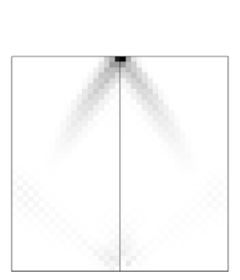

(e) $\gamma^{(3 r d)}$

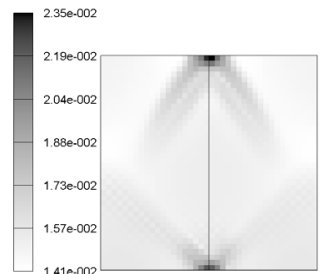

(b) $\rho_{S}^{(9 t h)}$

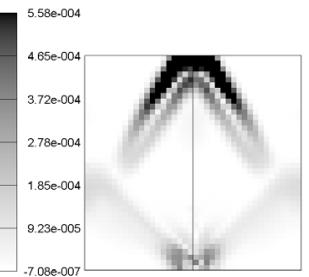

(f) $\left\|\rho_{G}^{(3 r d)}\right\|$

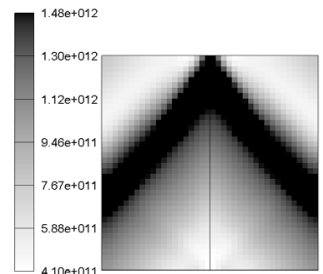

(c) $\rho_{G, \text { edge }}^{(9 t h)}$

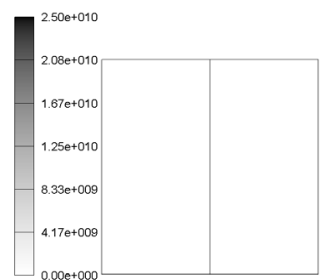

(g) $\gamma^{(10 t h)}$

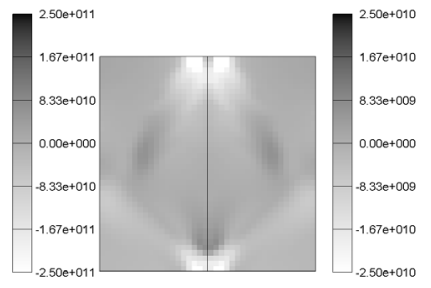

(d) $\rho_{G, s c r e w}^{(9 t h)}$

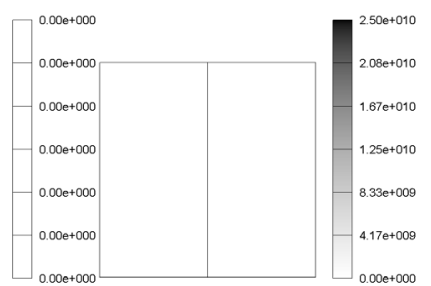

(h) $\left\|\rho_{G}^{(10 t h)}\right\|$

Fig. 8 (a) Distribution of plastic shear strain, (b) density distribution of statistically stored dislocations, (c) edge and (d) screw components of geometrically necessary dislocations on the primary slip system, (e), (g) distribution of plastic shear strain and (f), (h) density of norm of geometrically necessary dislocations on (111)[10 $\overline{1}]$ and $(1 \overline{1} 1)[110]$ slip systems when the average tensile strain $\bar{\varepsilon}_{y y}$ is $1 \%$ at the bicrystal model of $\alpha=46^{\circ}$. The unit of dislocation density is $\mathrm{m}^{-2}$

By the influence of the local strain hardening accompanying the activation of the secondary slip system resulting from the structure formation of the "GN dislocation band" in the primary slip system, the non-uniformity of slip deformation in the primary slip system develops and a new deformation field is formed. In accordance with the formation of the 
new deformation field, a new GN dislocation structure is formed both in the primary slip system and in the secondary slip system. Due to the dislocation interaction, a new local strain hardening also occurs. By the repetition of the series of deformation mechanisms, the aspect of the deformation field, after the secondary slip system is activated, is updated simultaneous with the advancement of deformation. For this reason, the difference of the state of deformation depending on the crystal orientation becomes remarkable. In particular, the difference in distribution of $\rho_{S}^{(9 t h)}, \rho_{G, \text { edge }}^{(9 t h)}$, and $\rho_{G, \text { screw }}^{(9 t h)}$ in the primary slip system is remarkable (Figs. 7(b) to (d), Figs. 8(b) to (d)).

From the result described above, it has been found that when the "GN dislocation band" in the primary slip system is formed in a high density, the secondary slip system is activated in the local area, and due to the effect of the dislocation interaction, the mean free path of the moving dislocation is reduced, local strain hardening occurs, and non-uniformity of the state of deformation develops.

\section{Conclusion}

The relationship between the density of GN dislocations that form the "GN dislocation band" and the value of the Schmid tensor, and the influence of the "GN dislocation band" imposed on the after-deformation state, were obtained by performing crystal plasticity analysis of tensile deformation of symmetric type bicrystals. The following results were obtained.

(1) The "GN dislocation band" occurs in the primary slip system accompanying the tensile of symmetric type bicrystals when the change in shape of the crystal grain due to the slip in the primary slip system is constrained (due to boundary conditions such as grain boundary and load condition), and the density of GN dislocations that accumulate in the "GN dislocation band" can be evaluated from the value of the Schmid tensor that determines the increment of the constrained plastic strain.

(2) As in the "GN dislocation band," when the GN dislocation structure in which the GN dislocations accumulate in a high density in a local area is formed, the secondary slip system is activated. Although the plastic shear strain that occurs in the secondary slip system is only $1 / 1,000$ to $1 / 100$ of the plastic shear strain that occurs in the primary slip system, a steep spatial gradient occurs because the strains occur in the local area and the GN dislocations accumulate in a high density.

(3) The state of deformation after the "GN dislocation band" in the primary slip system is formed in a high density and the secondary slip system is activated, due to the dislocation interaction between the primary slip system and the secondary slip system, the mean free path of the moving dislocation is reduced, the SS dislocations accumulate locally, local strain hardening in the primary slip system occurs, and non-uniformity of deformation develops abruptly.

\section{References}

(1) Ashby M. F., The Deformation of Plastically Non- homogeneous Alloys, Phil. Mag., Vol. 21 (1970), pp. 399-424.

(2) Hook, R. E. and Hirth, J. P., The Deformation Behavior of Isoaxial Bicrystals of Fe-3\% Si, Acta Metall., Vol. 15 (1967), pp. 535-551.

(3) Livingston, J. D. and Chalmers, B., Multi Slip in Bicrystal Deformation, Acta Metall., Vol. 5, No. 6 (1957), pp. 322-327.

(4) Hauser, J. J. and Chalmers, B., The Plastic Deformation of Bicrystals of F.C.C. Metals, Acta Metall., Vol. 9, No. 9 (1961), pp. 802-818.

(5) Kondou R. and Ohashi T., Crystal Plasticity Analysis of Non-uniform Deformation in Symmetric Type Bicrystals under Tensile Load and Formation of Geometrically Necessary Dislocation Bands, Journal of Solid Mechanics and Materials Engineering, Vol. 1, No. 1 
(2007), pp.114-125.

(6) Ohashi T., Numerical Analysis of Deformation Band Evolution and Sub-Macroscopic Stress Disorder in FCC Single Crystals, J. Japan Inst. Metals, (in Japanese) Vol. 53, No. 3 (1989), pp. 290-295.

(7) Ohashi T., Meso-scale Perspectives in the Analysis of Crystal Slip Deformation and Dislocation Accumulation, Trans. JSME, A, (in Japanese) Vol. 68, No. 675 (2002), pp. 1490-1497.

(8) Hill R., Generalized Constitutive Relations for Incremental Deformation of Metal Crystals by Multislip, J. Mech. Phys. Sol., Vol. 14 (1966), pp. 95-102.

(9) Ohashi T., Numerical Modeling of Plastic Multislip in Metal Crystals of F.C.C. Type, Phil. Mag. A, Vol. 70, No. 5 (1994), pp. 793-803.

(10) Ohashi T., Finite-Element Analysis of Plastic Slip and Evolution of Geometrically Necessary Dislocations in F.C.C. Crystals, Phil. Mag. Lett. Vol. 75, No. 2 (1997), pp. 51-57.

(11) Ohashi T., A New Model of Scale Dependent Crystal Plasticity Analysis, IUTAM Symposium on Mesoscopic Dynamics of Fracture Process and Materials Strength (2004), pp. 97-106.

(12) Ohashi T., Prediction of Macroscopic Mechanical Response of Metallic Materials Through Evaluation of Deformation Processes in Microstructure, The Iron and Steel Institute of Japan, 180 181 Nishiyama Anniversary Technique Lecture (in Japanese) (2004), pp. 73-95. 